\title{
Towards Using Value Tensions to Reframe the Value of Data Beyond Market-based Online Social Norms
}

\author{
Anisha T J Fernando \\ Lecturer, SAIBT \\ University of South Australia \\ Kathleen Lumley College \\ anisha.fernando@unisa.edu.au \\ Lesa Scholl \\ Head of College \\ Kathleen Lumley College
}

\section{Abstract}

Making sense of data, its value, and impact is imperative for individuals, organisations, and societies to function in the cyber age. The online interactions through which data flows present many benefits. However, the consumption of data and its value is problematic due to an overreliance on market norms as a substitute for values-based online social norms and practices, creating value tensions. Understanding the implications of data is further complicated due to the complex contextual nature of online interactions. These challenges are addressed through efforts from technology organisations and policy initiatives. Largely absent from these efforts is an understanding of the values needed to ground healthy online social interactions, and processes that nurture and afford the practice of these values in contextual community settings. Value tensions as an ethics tool can surface and clarify these interpersonal needs in understanding data and its impact, while its exploration can be grounded by the value sensitive design methodology. Communities may be appropriately placed to grapple with these value tensions given the contextual nature of interactions and the collective benefits and harms at stake. This discussion paper presents a preliminary research agenda raising questions on uncovering value tensions and understanding the values at stake to transform data practices and develop healthy online social norms, to reframe the value of data beyond market-based online social norms.

Keywords: Value Sensitive Design, Value Tensions, Social Values, Online Social Norms, Data Flows, Data Literacy, Data Ethics.

\section{Introduction: Purpose and Context}

Online interactions are fundamental to $21^{\text {st }}$ century life and offer many benefits for people and organisations alike such as effectiveness, efficiencies, convenience, and cost-savings. These interactions and behaviours are influenced by online social norms such as: checking Facebook or Instagram while on the bus, at work or when interacting with others; or, asking Google for instant answers when mildly curious. These pervasive activities may be harmless, but people seem to trust the information consumed even when it is misinformed (Thorson 2016). These new sources of data diets indicate a growing cultural trend where people may prefer consuming information via search engines over the need to access specialist knowledge sources.

By contrast, these activities have non-transparent and hidden possible traps of engaging online, unless values ground these interactions. For example, decisions can be made with 
misleading or misinformed non-specialist opinions presented as facts (Thorson 2016). Algorithms controlling the visibility and access to information may be primarily incentivised to generate monetary value rather than evaluate the trustworthiness of information. Recent changes made to Google's algorithms to focus on expertise, authoritativeness and trustworthiness measures for YMYL (Your Money Your Life) websites (i.e. content such as legal, financial, health-related etc. which may impact satisfaction and well-being of information consumers), indicate plausible risks and underscore the importance of healthy information consumption (Ray 2019).

In this context, values refer to social values, i.e. moral guiding principles of life and motivating behaviours practiced by people. These values and practices impact decisions people take in the pursuit of how to live well, shaping their identities and beings. The nature of the roles played by people in various societal units such as families, communications and organisations are influenced by these social values (Bruce 1999).

When people use technology, their values and practices are mediated by technology and its design (Verbeek 2011). Decisions people take in socio-technical contexts are technomoral choices shaped by the affordances of technologies (Vallor 2018). These values do not exist in isolation, but underpin online interactions. Value Sensitive Design (VSD) is a methodology which considers the values at stake when designing technology. In the VSD context, a value is "what is important to people in their lives, with a focus on ethics and morality" (Friedman \& Hendry 2019 p. 24). Value tensions, a VSD tool, offers an opportunity to consider values that may be in contrast, but hold importance in its interconnected relationship (Friedman \& Hendry 2019). The specific value tension that is pertinent to this discussion on understanding data is the tension between market- and social-based norms at play through online interactions. Given the focus on social values and the collective nature of its impact, the scope, and notions of values in the context of this exploration is at the broader societal level.

Within popular media and the broader society, there are questions around the ways in which online interactions are challenging established traditional family, cultural, or social values. Conventionally social norms are established through interactions in social structures such as families, communities, schools, but technology-based spaces present a new dynamic. Social norms in these spaces are mediated by technology (Verbeek 2011).

Values embedded in the design of technology may afford certain practices, influencing the establishment of social norms. General societal cues that signpost behaviour such as body language and practices like accountable relationships with people are not as visible in the online sphere. For example, the notice and consent approach or the default opt-in approach used to express privacy expectations on online websites does not recognise that people may not completely understand the details involved when consenting to use of the website and to transfer of their personal data (Nissenbaum 2010). Valuing data through the lens of marketbased norms may drive and increase the appetite for monetary value, risking the social nature and the integrity of online interactions.

Every time people are online, they generate data, use data, leak data, make decisions based on data and trust data. This data diet is increasingly becoming a frenzy-driven, adrenalin-fuelled activity where individuals seem to have the freedom to act through this lens (Eyal 2014). Such behaviour is often devoid of traditional norms such as respect for others or norms get drowned out or lost. Then what becomes easier and transparent are outrageous morally unacceptable 
behaviours experienced online such as trolling and cyber-bullying, and the normalising of carefully curated social worldviews leading to even Instagram or Twitter-driven suicides.

Other subtle unacceptable behaviours people think they can get away include photoshopping, creating deep fakes, manipulating other people's data to obscure the truth, hacking attractive honeypots, and social engineering sophisticated phishing campaigns to target people with poor awareness and data literacy skills. For example, comparing different notions of value where norms are distorted: creating and concealing fake money is forgery and money laundering, which are prosecutable crimes; while creating actual fake news to fuel misinformation or disinformation campaigns breaks social contracts, harms the integrity of interactions, and diminishes trust, but is usually dismissed as the Internet's next evolving fad. The boundaries between socio-technical practices that are considered appropriate and inappropriate are becoming blurred.

Hence, the consumption of data and its value, however, is problematic because people are more likely to propagate polarised views, are exposed to misinformation and untrustworthiness of sources, encounter disinformation campaigns, and experience effects of social isolation, mistrust and technology addiction (Policy Department for Citizens' Rights and Constitutional Affairs 2019; Badawy, Lerman \& Ferrara 2018; Pasquale 2015; Center for Humane Technology 2019; Courtwright 2019). Current efforts by organisations and governments are largely framed from an institutional standpoint, whereas data ethics issues usually affect individuals personally. Institutions are seen to not take accountability, while individuals are absolved of their responsibility to take action due to the siloed nature of sociotechnical interactions. For example, the ethical impacts of big data use and the complex nature of organisational needs are well documented (boyd \& Crawford 2012; Crawford, Miltner \& Gray 2014). Community-based initiatives may reinvigorate exploration of the values and the value tensions at play in relation to societal needs.

This discussion paper proposes the need to rethink or reframe the value of online data and the need to explore possibilities to encourage people to create healthy online spaces and practices using value tensions as an ethics tool to aid this process in community settings. Examples of common online interactions are used to describe market-based online social norms experienced and arguments are presented to empathise the need for online social norms, need to also be rooted in social values to sustain healthier ways of interacting online.

There is a need to uncover a deeper understanding of how surfacing this value tension can help people understand the contextual nature of their online interactions and how social values are formed and practice. This exploration of value tensions as an ethics tool can also provide useful input to designers of these technologies in imbuing ethical social values in technologies and mediating healthier online social norms.

\section{Motivation and Impact: Understanding the Value Tension at Stake - Social vs. Market based Online Norms}

In this section, the specific value tension of social vs. market norms influencing online interactions and the subsequent impact of data and its value is discussed. An overreliance on market-based online social norms has the potential to disrupt the integrity of people's online interactions. In this cyber age, market incentives drive data creation and use. Technology organisations are heavily influenced by advertising, where data is monetised. This data is freely acquired from people's interactions with proprietary technology, and its complexity is 
oblivious to the everyday person. Monetary value is manifested in the aggregation of data and the creation of behavioural surplus (Benkler 2006; Ariely 2008; Zuboff 2019). These technology organisations face competing incentives, where their strategic business goals compete with their stated intentions for ethical practices. Social norms will not hold in a market exchange because these are not grounded in social values (Ariely 2008).

For example, through social production, data is freely created by people in exchange for online services (Benkler 2006). The value of data (i.e. information goods in this context) appreciates with time and increases with aggregated use compared to traditional market goods that depreciate over time (Ariely 2008). The authorship of content is lost, and people are not rewarded in a typical market-based version of social production (Lanier 2014). It is harder for technology organisations to verify the trustworthiness of data when the incentive is to publish fast with sensationalism because these indicators drive user clicks, which drive advertising revenue and increase market-based value. Traditional gatekeepers are replaced or devalued, and efforts to introduce gatekeepers, as done by Facebook, fall short because within this new medium the norms and impacts are in flux and the rate of data production is more than what is manageable by a single entity regardless of its size (Zuboff 2019). Hence, organisations are not best placed to foster nurturing healthy online social norms because their structures may not lend to effective accountability (as seen in the debate to define the big technology organisations as media or technology companies).

These market-based online social norms propagate data being valued in monetary terms, driven by market incentives. For example, Facebook generated US55.8 billion in revenue for 2018 , using people's attention, data and interactions as raw materials in this data processing chain that drives surveillance capitalism (Statista 2019; Zuboff 2019). Established information providers use advertising to drive network effects, reduce operating costs and enable greater efficiency. This framing is risky, as data communicates details about people - it reveals our identities and preferences; our personas become public sources with limited control and open to influencing. The value of data is being framed as mostly transactional and lies in aggregation from a market-based perspective. Statistically significant inferences guide the development of online interactions like personalised search results. It is not profitable to solely target an individual person's preferences and habits with higher degrees of precision. A person's identity is intertwined with their experiences. Data is also human, containing characteristics about people. Is the social association with data about people and their connections lost when viewing it through this marked-based frame?

People ascribe meaning to information derived based on data processed through these interactions (Thorson 2016, Casanovas et al. 2017; Dawson et al. 2019). Through these misplaced intentions and unethical practices in creating and releasing data, value is placed on data by external systems. This absence of adequate agency by people leaves vulnerabilities for data misuse and misinterpretation. This distorted, manipulated, and fictious data accumulates value through interactions and sharing as opposed to value created because of its reliability, credibility, and trustworthiness (Pasquale 2015; Zuboff 2019).

These market-based norms add to the complexity of decision-making involved in online interactions. For example, consenting to privacy is termed conventionally as trade-offs exploring the nature and the extent to which people are willing to release personal data for benefits (financial or otherwise). However, humans are cognitively limited in their ability to make complex decisions such as the decisions required in online privacy (Ariely 2008). Low 
levels of technical literacy and aliteracy inhibit contextual decision-making in online contexts (Naughton 2012). Technical literacy concerns the levels of awareness and understanding around risks associated with data flows. Aliteracy occurs when people have the ability to understand but are not interested or find it difficult to convey their expectations due to complexities in implementing and using alternate mechanisms. For example, a survey of Australian consumers reported that people found it difficult to distinguish the provenance and operation of different elements of a search engine results page, particularly with integrated vertical search services and less so with distinguishing ads from search results (Scardamaglia \& Daly 2016).

The disconnect between social and market values around technology innovation triggers a norm, where the market value take precedence over the social value. Traditionally, human interactions are premised on social values. In online interactions, social values will not hold in a market exchange because the interaction is not premised on social values, but largely influenced by market-based values (Ariely 2008). For example, standardised testing introduced mechanisms of measuring student performance in education. However, it has become the de-facto measure of student learning, where the incentive is to perform better on tests as opposed to creating a stable culture focused on learning where performances achieving good learning outcomes will naturally emerge (Ariely 2008). Both students and schools are measured against this indicator, which influences their roles in a learning context, which is usually termed as a social activity.

The effects of social values, whether positive or negative, impact human beings, not technology. Social cohesiveness is lost in these market-based online exchanges. Market-based norms and values stems from commercial motivations. The cost of interactions is considered free by people, largely influenced by the ethos stemming from academic activities that kickstarted the development of the internet. Being on the internet is viewed as a social exchange. For example, on social media, friendships are measured by the amount of likes a photo gets or the traction a post gets. These measures of association show a quantitative dimension of popularity, while the qualitative sentiments are lost, losing that richness and depth normally associated with friendships. People are accustomed to encountering new functionality and form new habits through their online interactions. People may be incentivised to provide inaccurate information to avoid loss of social values like privacy and such falsification of data is a dis-benefit to organisations because data quality diminishes too. Market-based norms tend to commodify social values, whereas people see services as being free on the Internet.

Is there a place for social values in a medium driven by market norms? For example, the Country Fire Service organise themselves around a community purpose for safety and wellbeing. In open source software development, many amateur and expert programming enthusiasts spent countless hours problem-solving and helping each other out. These communities are widely popular and sustain themselves without any significant market incentive, and prioritise social values over market-based values. Community-facilitated initiatives may help people make sense of data, its value and impact beyond experiencing and habituating to largely market-based values and norms. A community setting can make visible the importance of protecting social values and the integrity of online interactions in technology-mediated spaces. The benefits and harms imbued by technology are not just limited to individuals, but have a collective societal impact and scope. 


\section{Current Efforts and New Challenges}

Initiatives to address making sense of data, its value and impact from an ethical lens in the cyber age focus mainly on technological and legal perspectives. Technology initiatives which include efforts to create empathetic and ethically minded technologies are growing, for example, educational programs focus on equipping technologists and IT professionals with data ethics principles (Massachusetts Institute of Technology 2019). These efforts are codified in organisational settings through professional codes of conduct, policies, and data ethics pledges. These professionals are the focus of educational efforts because their actions in their professional roles determine how personal data of people is used. Such education is focused on how organisations can ethically use the data for the purpose for which it is collected. Even in this organisational context, questions arise in terms of vested competing interests, with commercial market incentives influencing ethical practices and shaping online social norms.

Other initiatives addressing online ethics are policy programs and legal reframing. Visible policy mechanisms include EU regulations around legal compliance measures to protect data across transnational boundaries, creating independent ethics advisory boards, training programs; antitrust measures and lawsuits (Casanovas et al. 2017; Dawson et al. 2019; Stiglitz 2019). Efforts to divide large technological companies are suggested as they act as monopolies with disproportionate market effects, creating power and information asymmetries (Stiglitz 2019; Zuboff 2015; Nissenbaum 2010).

While expected behaviours and appropriate values to be upheld are codified for IT professionals in organisational or societal bodies through codes of conduct, nothing equivalent exists for appropriate norms and data practices for individuals and members of communities. It is unclear how online social values and norms are nurtured given the strong influence of technology-mediation in interactions (Verbeek 2011). Tools are extensions of self and afford us value in the act of interacting (Gibson 1979; Heidegger 1977). If the core value is not embedded in interactions, the value cannot be exercised. Individuals who use these technologies are absolved from their personal responsibility because of the isolating, siloed, contextually-public nature of online interactions.

Missing from this discourse is a discussion around community-centred initiatives to address these challenges. A person's data is about that individual. Often the best forms of privacy protection or effectiveness of ethical practices is in the act of data creation, because this is where the risk is at a minimum. It is important to develop proactive data protection initiatives because privacy loss is often instantaneous and reputational, and may affect more than just an individual, given its interconnected nature. While advertisers need to take responsibility for the secondary collection of data and could benefit from education around incentives and ethical practices, community education initiatives are also needed. There is a lack of community initiatives to teach people basic data skills and data ethics practices to nurture and guide their socio-technical interactions.

\section{Conclusion: Future Steps and Research Agenda}

Given that data consumption is engineered, and unhealthy online social norms and its effects are becoming more evident in society, the authors propose a preliminary research agenda to address these questions:

- What are the values at stake in different market-influenced online contexts? 
- What can be done to reframe the valuing of data and transform data practices?

- What can be done to develop healthy online social norms?

To address these questions, the proposed research methodology needs to be cognisant of the values at play. Value sensitive design (VSD) considers the application of human values in the design of technology through a rigorous process (Friedman, Kahn \& Borning 2009).

Value tensions as a VSD-based ethics tool could help unpack contrasting values of data influenced largely by market norms and offer opportunities to ask how social values may help reframe or understand data and its value. This work grounded in VSD, would contribute towards extending current understandings of online social values and norms, and would explore how broader societal structures are impacted by these socio-technical value tensions.

Future work would include conducting a systematic literature review on the application of value tensions as an ethics tool to identify mechanisms through which value tensions was prototyped. Following this conceptual exploration, the proposed value tensions tool will be co-designed with community-based participants and rapidly prototyped to create network learning resources which will propagate understanding data, reframing its value, and surface social values for online interaction contexts. Investigations exploring these questions underpinned by VSD will provide a useful lens through which to study the value tensions driven by social vs. market-based norms in online contexts.

Unhealthy online social norms driven by market incentives influence people's values and practices around data. For example, misinformation propagated by algorithms incentivised by monetary value risks the trustworthiness of information and the integrity of the interactions through which people consume information. Market-based norms create value tensions with the social nature of online interactions. Value tensions as an ethics tool is proposed as a method to facilitate an understanding of data and the social values at stake in online contexts through community-based initiatives. It is important to understand how healthy online social norms, practices and values can be established to reframe the value attached to data and sustain the integrity of socio-technical interactions.

\section{Acknowledgements}

The authors would like to thank Joseph Hall for discussions on the early versions of this paper for the AiCE 2019 Conference, and Monica Behrend for stimulating critique and reviewing early drafts. The authors would also like to thank the anonymous reviewers who have taken the time to review and provide insightful comments that helped develop this paper.

\section{References}

Ariely, D. (2008). Predictably irrational: the hidden forces that shape our decisions. New York: HarperCollins Publishers Ltd.

Badawy, A., Lerman, K., \& Ferrara, E. (2018, August). Who Falls for Online Political Manipulation? Retrieved from https://arxiv.org/abs/1808.03281

Benkler, Y. (2006). The wealth of networks: how social production transforms markets and freedom. New Haven, Connecticut Yale: University Press.

boyd, D., \& Crawford, K. (2012). Critical questions for big data: Provocations for a cultural, technological, and scholarly phenomenon. Information, Communication E Society. 15(5), 662-679. 
Bruce, S. (1999). Sociology: A very short introduction. Great Britain: Oxford University Press.

Casanovas, P., De Koker, L., Mendelson, D., \& Watts, D. (2017). Regulation of Big Data: Perspectives on strategy, policy, law and privacy. Health and Technology, 7(4), 335-349.

Center for Humane Technology. (2019, June). The problem: the extractive attention economy is tearing apart our shared social fabric. Retrieved from https://humanetech.com/problem/

Courtright D. T. (2019). The age of addiction: how bad habits become big business. Cambridge, Massachusetts: The Belknap Press of Harvard University.

Crawford, K., Gray, M., \& Miltner, K. (2014). Big Data - Critiquing Big Data: Politics, Ethics, Epistemology - Special Section Introduction. International Journal of Communication. 8(10), $1663-1672$.

Dawson D., Schleiger E., Horton J., McLaughlin J., Robinson C., Quezada G., Scowcroft J., \& Hajkowicz S. (2019, April). Artificial Intelligence: Australia's Ethics Framework. Data61 CSIRO, Australia. Retrieved from https://data61.csiro.au/en/Our-Work/AI-Framework

Policy Department for Citizens' Rights and Constitutional Affairs. (2019, February), Disinformation and propaganda-impact on the functioning of the rule of law in the EU and its Member States. European Parliament. Retrieved from https://www.europarl.europa.eu/RegData/etudes/STUD/2019/608864/IPOL STU(2019) 608864 EN.pdf

Eyal N. (2014). Hooked: how to build habit-forming products. London, England: Penguin.

Friedman, B., Kahn, P. \& Borning, A Himma, K. (2009). Value Sensitive Design and Information Systems. In K.E. Himma \& H.T. Tavani (Eds) The Handbook of Information and Computer Ethics (pp. 69-101). Hoboken, NJ, USA: John Wiley \& Sons.

Friedman, B. \& Hendry, D. G. (2019). Value Sensitive Design: Shaping Technology with Moral Imagination. Cambridge, MA: MIT Press.

Gibson, J. (1979). The ecological approach to visual perception: classic edition. Boston, Massachusetts: Psychology Press.

Heidegger, M. (1977). The question concerning technology and other essays. New York: Harper \& Row.

Lanier, J. (2014). Who owns the future? London, England: Penguin Books Ltd.

Massachusetts Institute of Technology. (2019). Professional Education - Ethics of AI: Safeguarding Humanity. Retrieved from https://professional.mit.edu/programs/shortprograms/ethics-ai

Naughton, J. (2012). What you really need to know about the Internet: From Gutenberg to Zuckerberg. Great Britain: Quercus.

Nissenbaum, H. (2010). Privacy in context, Stanford, California: Stanford University Press.

Pasquale, F. (2015). The black box society: the secret algorithms that control money and information. Cambridge, Massachusetts: Harvard University Press.

Ray, L. (2019). Google's algorithm undergoes enormous shifts to prioritise content focused on expertise, authoritativeness and trustworthiness. Journal of Digital $\mathcal{E}$ Social Media Marketing. 7(2), 128-136. 
Scardamaglia, A. \& Daly, A. (2016). Google, online search and consumer confusion in Australia. International Journal of Law and Information Technology. 24 (3), 203-228.

Statista. (2019). Facebook's annual revenue and net income from 2007 to 2018 (in million U.S. dollars). Retrieved from https://www.statista.com/statistics/277229/facebooks-annual-revenueand-net-income/

Stiglitz J.E. (2019). People, power and profits: progressive capitalism for an age of discontent. Great Britain: Allen Lane, Penguin Random House UK.

Thorson, E. (2016). Belief echoes: the persistent effects of corrected misinformation. Political Communication. 33(3), 460-480, Retrieved from doi: 10.1080/10584609.2015.1102187

Vallor, S. (2018). Technology and the virtues: A philosophical guide to a future worth wanting. New York: Oxford University Press.

Verbeek, P. P. (2011). Moralizing technology: understanding and designing the morality of things, Chicago: University of Chicago Press.

Zuboff, S. (2015). Big other: Surveillance capitalism and the other prospects of an information civilization. Journal of Information Technology, 2015(30), 75-89.

Zuboff, S. (2019). The age of surveillance capitalism: The fight for the future at the new frontier of power. New York: Public Affairs.

Copyright: (C) 2020 Fernando \& Scholl. This is an open-access article distributed under the terms of the Creative Commons Attribution-NonCommercial 3.0 Australia License, which permits non-commercial use, distribution, and reproduction in any medium, provided the original author and AJIS are credited.

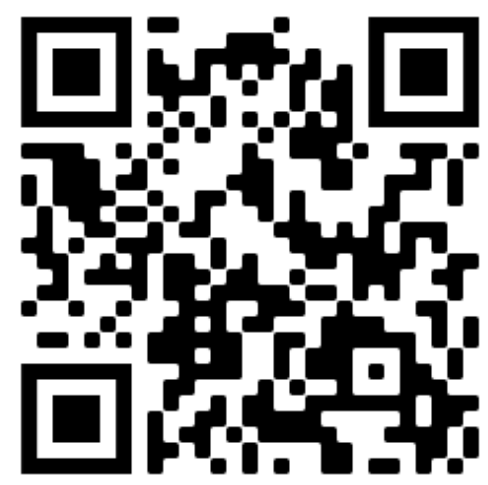

